\title{
Co-injection of mesenchymal stem cells with endothelial progenitor cells accelerates muscle recovery through an endoglin-dependent mechanism
}

Elisa Rossi ${ }^{1,2}$, Céline Goyard ${ }^{1,2,3}$, Audrey Cras ${ }^{1,2,4}$, Blandine Dizier ${ }^{1,2}$, Nour Bacha ${ }^{1,2}$, Anna Lokajczyk $^{1,2}$, Coralie L Guerin ${ }^{5}$, Nicolas Gendron ${ }^{1,2,8}$, Benjamin Planquette ${ }^{1,2,3}$, Virginie Mignon $^{1,6}$, Carmelo Bernabeu ${ }^{7}$, Olivier Sanchez ${ }^{1,2,3}$ and David M Smadja ${ }^{1,2,8}$

${ }^{1}$ Université Paris Descartes, Sorbonne Paris Cité, Paris, France

${ }^{2}$ Inserm UMR-S1140, Paris, France

${ }^{3}$ AP-HP, European Georges Pompidou Hospital, Respiratory Medicine Department, Paris, France

${ }^{4}$ AP-HP, Saint Louis Hospital, Cell Therapy Department, Paris, France

${ }^{5}$ National Cytometry Platform, Department of Infection and Immunity, Luxembourg Institute of Health, Luxembourg

${ }^{6}$ Cellular and Molecular Imaging Facility, Inserm US 25, CNRS UMS 3612, Faculty of Pharmacy of Paris, Paris Descartes University

${ }^{7}$ Centro de Investigaciones Biológicas, Consejo Superior de Investigaciones Científicas (CSIC), and Centro de Investigación Biomédica en Red de Enfermedades Raras (CIBERER), Madrid, Spain

${ }^{8}$ AP-HP, European Georges Pompidou Hospital, Hematology Department, Paris, France

\section{Address for correspondence:}

\section{Prof. David Smadja}

European Hospital Georges Pompidou, Hematology Department and UMR-S1140

20 rue Leblanc, 75015 Paris, France, Tel: +3156093933, Fax: +3156093393

E-mail: david.smadja@aphp.fr 


\section{ABSTRACT}

Endothelial colony-forming cells (ECFCs) are progenitor cells committed to endothelial lineages and have robust vasculogenic properties. Mesenchymal stem cells (MSCs) have been described to support ECFC-mediated angiogenic processes in various matrices. However, MSC-ECFC interactions in hind limb ischemia (HLI) are largely unknown. Here we examined whether coadministration of ECFCs and MSCs bolsters vasculogenic activity in nude mice with HLI. In addition, as we have previously shown that endoglin is a key adhesion molecule, we evaluated its involvement in ECFC/MSC interaction. Foot perfusion increased on day 7 after ECFC injection and was even better at 14 days. Co-administration of MSCs significantly increased vessel density and foot perfusion on day 7 but the differences were no longer significant at day 14. Analysis of mouse and human CD31, and in situ hybridization of the human ALU sequence, showed enhanced capillary density in ECFC+MSC mice. When ECFCs were silenced for endoglin, coinjection with MSCs led to lower vessel density and foot perfusion at both 7 and 14 days $(\mathrm{p}<0.001)$. Endoglin silencing in ECFCs did not affect MSC differentiation into perivascular cells or other mesenchymal lineages. Endoglin silencing markedly inhibited ECFC adhesion to MSCs. Thus, MSCs, when combined with ECFCs, accelerate muscle recovery in a mouse model of hind limb ischemia, through an endoglin-dependent mechanism. 


\section{What is known about this topic?}

- Critical limb ischemia (CLI) is the most severe stage of peripheral arterial disease (PAD). A therapeutic approach based with vasculogenic cells has given promising results.

- Endothelial colony-forming cells (ECFCs) are progenitor cells committed to endothelial lineages and have robust vasculogenic properties.

- Mesenchymal stem cells (MSCs) have been described to support ECFC angiogenic processes in various matrices. Interactions between MSCs and ECFCs in hind limb ischemia (HLI) are largely unknown.

\section{What does this paper add?}

- We describe beneficial cooperation between MSCs and ECFCs in hind limb ischemia recovery, with an increase in blood flow and less necrosis than after ECFC injection alone.

- Silencing of endoglin in ECFCs abolishes this MSC-ECFC interaction in the HLI model, with decreased vessel density, foot perfusion and muscle incorporation of human endothelial cells.

- Endoglin silencing in ECFCs did not affect MSC differentiation into perivascular cells or other mesenchymal lineages, but it markedly decreased ECFC adhesion to MSCs.

- MSC coinjection with ECFCs accelerates HLI muscle recovery through an endoglindependent mechanism. We propose the systematic use of MSCs to improve ECFC engraftment in hind limb ischemia. 


\section{INTRODUCTION}

Critical limb ischemia (CLI) is the most severe stage of peripheral arterial disease (PAD) and is associated with a high risk of amputation and death despite surgical bypass and endovascular procedures. Therapeutic angiogenesis based on cell therapy has given promising results and may be suitable for patients who are not candidates for revascularization $(1,2)$. In 2002, TateishiYuyama et al. (3) published a randomized, controlled pilot trial of autologous non-cultured bone marrow-derived mononuclear cells (BM-MNCs) in CLI patients. Several different bone marrow cell (BMC) types, numbers and routes of administration have since been used, but the mechanisms underlying the benefits associated with BMC therapy remain poorly understood. Newly formed vessels have been observed after BMC therapy in CLI (4-6), but their cellular origin is unclear. The cells most likely to give rise to vessels are endothelial progenitor cells and particularly a subtype isolated from peripheral blood, namely endothelial colony-forming cells (ECFCs). ECFCs are precursors committed to the endothelial lineage. They are clonogenic and have robust vasculogenic properties $(5,7)$. However, their small numbers and poor expansive properties when isolated from human adults limit their use as an autologous cell therapy product $(2,8)$. Strategies to improve the therapeutic potential of ECFCs are therefore needed. Here we tested a two-cell strategy, using human ECFCs and human mesenchymal stem cells (MSCs), to induce functional, perfused vascular networks. This approach has previously been tested in provisional extracellular matrices (9), but not in vivo.

Endoglin (CD105) is an RGD-containing glycoprotein of $180 \mathrm{kDa}$ predominantly expressed in endothelial cells and playing a critical role in the cardiovascular system (10). We recently reported that endoglin regulates the adhesion of mural cells (11) and inflammatory leukocytes (12) to endothelial cells. The aim of the present study was to assess the effect of ECFC and MSC 
co-administration in terms of vascular formation in a model of induced hindlimb ischemia, and to analyze the effect of endoglin on ECFC/MSC vasculogenic properties during ischemic leg revascularization. 


\section{MATERIALS AND METHODS}

\section{ECFC and MSC isolation and culture}

ECFCs were isolated from the adherent mononuclear cell (MNC) fraction as described in (5). ECFCs were then expanded on fibronectin (FN)-coated plates $(1 \mu \mathrm{g} / \mathrm{cm} 2$; Millipore, Billerica, MA, USA) using EGM-2 (without hydrocortisone; Lonza, Walkersville, MD, USA) supplemented with $20 \%$ fetal bovine serum (FBS; Hyclone, Logan, UT, USA). Mesenchymal stem cells (MSCs) were isolated from the mononuclear cell (MNC) fraction of human adult bone marrow, as described in (13) and according to the International Society for Cellular Therapy (ISCT) standard criteria for MSC isolation and characterization (14). The phenotype of the MSC used here is described in supplementary Figure 1. ECFC and MSC were cultured separately in appropriate media to maintain their characteristics. Adherent cells were trypsinized before coinjection.

\section{Cell transfection with endoglin siRNA}

Endoglin-specific siRNA was used to silence human endoglin (endoglin; sc-35302, Santa Cruz Biotechnology, CA, USA) as previously described (11). Briefly, siRNA was mixed with the Dharmafect reagent (SO-2511539G Dharmacon, USA) at $10 \mu \mathrm{M}$ to obtain transfection complexes, which were added to ECFCs in EGM2 medium in six-well plates. ECFCs transfected with scrambled siRNA (Scramble, Allstars Neg. control siRNA, Qiagen, Cambridge, MA, USA) were used as a control (control-siRNA). To determine the efficiency of endoglin suppression, immunofluorescence flow cytometry was used to detect endoglin expression. Endoglin silencing over a seven-day period is shown in Supplementary Figures 2 and 3. 


\section{Hindlimb ischemia model, Doppler analysis and tissue collection}

Four- to five-week-old male athymic nu/nu mice underwent right femoral artery ligation under isoflurane anesthesia, as previously described (15). The left femoral artery was permanently ligated. The day after ligation, Laser Doppler Perfusion Imaging (LDPI) was used to investigate cutaneous blood flow. ECFCs +/- MSCs, or just PBS, were injected into the retro-orbital region. Seven and 14 days after ischemic ligation and injections of conditioned media, reperfusion of the ischemic limb was analyzed by LDPI. Briefly, the limbs were placed on a heating plate at $37^{\circ} \mathrm{C}$ to minimize temperature variations. Foot perfusion was measured with an LDPI device. Perfusion was expressed as the ratio of the ischemic to the non-ischemic foot paw. At 14 days, the gastrocnemius muscle was isolated, embedded in OCT and frozen at $-80^{\circ} \mathrm{C}$. Cryostatic sections were subjected to hematoxylin and eosin (H\&E) staining, immunostochemistry, and in situ hybridization. Mice $(n=24)$ were injected with PBS $(n=6)$, ECFC control siRNA (n=6), ECFC control-siRNA + MSCs at a ratio of 1:1 $(n=6)$, or ECFC endoglin-siRNA + MSCs at a ratio of $1: 1(\mathrm{n}=6)$.

We verified that cells were not retained in the retro-orbital sinus after injection.

ECFC and MSC distribution was monitored by using Fluorescence Molecular Tomography (FMT) (VisEn Medical, Perkin Elmer) as previously described by us and others (16-18). ECFC were labeled with Vivotag 750 (Perkin Elmer, Courtaboeuf, France), and MSC with Vivotag 680 (Perkin Elmer, Courtaboeuf, France) before injection (see fluorescence before injection in Supplementary Figure 4A). As shown in Supplementary Figure 4B, cells were detected in the retroorbital region just after injection, but no fluorescence was observed after 24 and/or 48 hours. Ten days after injection, bright near-infrared fluorescence was seen in the ischemic muscle, as we had previously observed (16), indicating cell homing. No fluorescent signal was seen in non- 
ischemic muscle. As shown in Supplementary Figure 4C, a significant fluorescent signal was detected by flow cytometry on isolated ischemic muscle cells, in contrast to non-ischemic muscle cells.

\section{Immunohistochemistry}

Frozen sections $(5 \mu \mathrm{m})$ were fixed with acetone at $-20^{\circ} \mathrm{C}$, rinsed in PBS and treated with $0.3 \%$ $\mathrm{H}_{2} \mathrm{O}_{2}$ in methanol for $20 \mathrm{~min}$ at room temperature (RT) to block peroxidases. After rinsing twice with PBS, a blocking solution (PBS, 10\% FBS, 0.2\% Triton X100) was applied for 30 min at RT. Then, a primary rat anti-mouse CD31 monoclonal antibody (BD, Bioscience) was added overnight at $4{ }^{\circ} \mathrm{C}$, followed by a biotin-labeled secondary anti-rat IgG antibody (Jackson Immuno Research). After washing with PBS, the sections were incubated for 45 min with the ABC kit (Vector), prior to exposure to diaminobenzidine (SigmaFast) for $5 \mathrm{~min}$. Counterstaining with hematoxylin was performed before treating the sections with Eukitt mounting medium (SigmaAldrich). Staining with mouse anti-human CD31 (BD Bioscience) was carried out as above, using biotin-labeled goat anti-mouse IgG as secondary antibody (Jackson Immuno Research).

\section{Necrosis analysis}

Muscle samples were processed for hematoxylin and eosin staining. The stained slides were analyzed by a researcher blinded to the experimental groups, using a light microscope at x10 and x20 magnification. Scores from 0 to 3 were attributed, based on the degree of cell damage (0 no damage, 3 most severe damage). Each slide was assessed twice. The slides were assessed according to cell shape, the number of neutrophils, and the degree of necrosis. In general, we could identify: 
1. Early-stage necrosis, with membrane damage at several regions of individual fibers. We found an association with clear regions in cytoplasm and histiocytic cells invade and replace muscle fibers. Necrotic muscle fibers.

2. Late muscle necrosis, where the fibers are invaded and replaced by phagocytes. Late phagocytic cells move out of necrotic muscle fiber space occupied by necrotic muscle fibers "Collapses". Some histiocytes may be present during muscle regeneration.

According to the literature (19), we defined a Grade 1 response as $<50 \%$ necrosis, a Grade 2 response as $50-80 \%$ necrosis, and a Grade 3 response as $>80 \%$ necrosis. Score 1 corresponded to $<50 \%$ necrosis (Grade 1). Supplementary Figure 5 illustrates normal, necrotic and regenerating myofibers.

\section{In situ hybridization (ISH) with digoxigenin-labeled Alu DNA probes}

DNA-DNA ISH was performed as previously described (20). After deparaffinization with ZZ Prep Standard and fixation for 32 min at $37^{\circ} \mathrm{C}$, we pretreated the slides with RiboClear for 12 min at $37^{\circ} \mathrm{C}$ and with $\mathrm{CC} 2$ Court with protease 3 for $20 \mathrm{~min}$. The Alu POS CTL probe was added by denaturation at $85^{\circ} \mathrm{C}$ for 8 min and hybridization was performed at $50^{\circ} \mathrm{C}$ for $1 \mathrm{~h}$. After washing the samples twice with $2.0 \mathrm{X} \mathrm{SSC}$ at $45^{\circ} \mathrm{C}$ for $4 \mathrm{~min}$, we applied RiboFix for $20 \mathrm{~min}$ at $37^{\circ} \mathrm{C}$ and biotin SP-conjugated mouse monoclonal IgG anti-FITC (Jackson Immunoresearch, 200-062-037; 1/200e) for 32 min at $37^{\circ} \mathrm{C}$. Using the ISH BlueMap XT detection kit, the signal was revealed for 1h and the slides were counterstained with Red Stain II for 4 min. 


\section{Cell adhesion assays}

Cultures of MSCs in 12-well plates were used in adhesion assays with ECFCs. When MSCs were confluent, control-siRNA ECFCs or endoglin-siRNA (endoglin siRNA) ECFCs, previously labeled with the fluorescent cell staining dye carboxyfluorescein succinimidyl ester (CFSE, CellTrackerTM, Life Technologies), were added (50 000 cells/well). After 30 minutes of incubation a $37^{\circ} \mathrm{C}$ with or without chemokines/growth factors $(100 \mathrm{ng} / \mathrm{mL} \mathrm{SDF} 1 \alpha$ or $50 \mathrm{ng} / \mathrm{mL}$ VEGF), the wells were rinsed with PBS 3 times and photographed with a fluorescence optical microscope (Observer D1, Zeiss) connected to a CCD camera (QIclick FCLR-12, Qimaging, Roper Scientific). The Image J program was used to quantify ECFC adhesion.

\section{MSC transdifferentiation}

Adipogenesis. MSCs were incubated in 6-well plates in adipogenic medium consisting of DMEM with $1 \mathrm{~g} / \mathrm{L}$ glucose supplemented with $20 \% \mathrm{FBS}, 0.5 \mathrm{mM}$ isobutylmethylxanthine (Sigma), $60 \mu \mathrm{M}$ indomethacin (Sigma) and $10^{-6} \mathrm{M}$ dexamethasone. The medium was replaced every 3-4 days for 14 days. After fixation with $4 \%$ paraformaldehyde (PFA), cells were stained for 30 min with $1 \mathrm{~mL}$ of $1 \mu \mathrm{g} / \mathrm{mL}$ Oil Red O (Sigma). Cells containing lipid vacuoles were observed/counted under the microscope.

Osteogenesis. MSCs were incubated in 6-well plates in osteogenic medium containing Dulbecco's Modified Eagle's Medium (DMEM) with $4.5 \mathrm{~g} / \mathrm{L}$ glucose (Life Technologies) supplemented with $10 \%$ FBS (Hyclone), $10^{-7} \mathrm{M}$ dexamethasone (Sigma), $50 \mu \mathrm{g} / \mathrm{mL}$ ascorbic acid (Sigma) and $3 \mathrm{mM} \mathrm{NaH}{ }_{2} \mathrm{PO}_{4}$ (Sigma). The medium was replaced every 3-4 days for 14 days. To measure alkaline phosphatase (ALP) activity, cells were fixed in methanol and stained for $10 \mathrm{~min}$ with 5-bromo-4-chloro-3-indolyl phosphate/nitro blue tetrazolium (BCIP/NBT) buffered 
substrate (Sigma). To assess calcium accumulation, cells were fixed in 4\% PFA and stained for 10 min with $1 \mathrm{~mL}$ of $40 \mathrm{mM}$ Alizarin Red (Sigma).

\section{Immunofluorescence studies}

Culture chamber slides were coated with gelatin and seeded with ECFCs control-siRNA+ MSCs or with ECFCs endoglin-siRNA+ MSCs at a 1:1 ratio. After co-culture for 7 days, MSCs differentiate into perivascular cells (11). Once differentiated, the cells were fixed with cold pure methanol on ice for $10 \mathrm{~min}$. For ECFC immunostaining, samples were incubated with a mouse antibody against human von Willebrand factor (Dako) for $1 \mathrm{~h}$ at room temperature, followed by the secondary antibody Texas Red anti-mouse IgG (Vector) for $1 \mathrm{~h}$ at RT. Differentiated MSCs were incubated with anti-human calponin (Abcam), anti-PDGFRb (Santa Cruz), anti-human NG2 (R\&D Systems), anti-human aSMA (Sigma) or a negative control antibody (Santa Cruz). After washing, samples were incubated with the appropriate FITC-labeled secondary antibody, nuclei were counterstained with TO-PRO-3 (642/661, Invitrogen) and samples were mounted in IBDI mounting medium.

\section{Statistical analysis}

Data are shown as means \pm SEM. Significant differences were identified by ANOVA followed by Fisher's protected least-significant difference test. Intergroup comparisons were based on the Mann and Whitney nonparametric test. All statistical tests were performed using the Stat View software package (SAS, Cary, NC). Differences with $P<0.05$ were considered statistically significant. 


\section{RESULTS}

\section{MSCs accelerate muscle recovery induced by ECFCs}

The effect of a single ECFC injection on angiogenesis in hind limb ischemia was compared to that of a single ECFC+MSC co-injection. Mice were injected with PBS $(n=6)$, ECFCs $(n=6)$ or ECFCs+ MSCs $(n=12)$ at a ratio of 1:1. Cutaneous blood flow was analyzed on days 1, 7 and 14. On day 7 , we observed increased revascularization after injection of ECFCs alone $(* \mathrm{p}=0.006$ vs PBS). MSC co-injection further improved revascularization on day 7 (*p=0.0001 vs ECFCs alone). On day 14 after injection, the increased revascularization in mice receiving ECFCs was still significant $(* \mathrm{p}=0.003$ vs $\mathrm{PBS})$, whereas no significant difference was observed between ECFCs and ECFCs/MSCs (p=0.34) (Figure 1).

On day 14, H\&E staining of the gastrocnemius muscle showed a high level of necrosis in the PBS group, as expected. ECFC injection significantly reduced necrosis $(* \mathrm{p}<0.0001)$, and co-injection of ECFCs and MSCs further reduced necrosis $\left({ }^{*} \mathrm{p}=0.01\right)$ (Figure $2 \mathrm{~A}$ and $\left.2 \mathrm{~B}\right)$. We found an increase in microvessel density in ECFC- and ECFC+MSC-treated mice, with no significant difference between the groups $(\mathrm{p}=0.53$, Figure $2 \mathrm{C})$, although the vessels in ECFC+MSC mice seemed larger. Incorporation of human cells in the mouse leg was demonstrated by hCD31 immunohistochemistry (Figure 2A, 2D) and by in situ hybridization of the human ALU sequence (Figure 2A). We found an increase in human vessel numbers in ECFCs and ECFC+MSC, with no significant difference between the groups ( $\mathrm{p}=0.21$, Figure 2D). As previously described (21), myofibrils appeared in regenerated tissues. Indeed, we found an increase in myofibers with central nuclei in the ECFC and ECFC+MSC groups. Our results suggest that co-injection of ECFC+MSC accelerates muscle recovery, with a better blood flow and less necrosis, but the same endothelial formation inside muscle at 14 days. 


\section{ECFC endoglin inhibition blocks the enhancement of muscle recovery induced by MSCs}

After testing the efficacy of endoglin silencing (Supplementary Figure 1), we injected endoglinsiRNA ECFCs + MSCs $(n=6)$ and compared their effects with those of control-siRNA ECFCs + MSCs (n=6) and PBS (n=6 mice). We analyzed cutaneous blood flow on days 1, 7 and 14 after injection (Figure 3). As expected, co-injection of control-siRNA ECFCs and MSCs improved cutaneous blood flow (Figure 3) and reduced necrosis (Figure 4A and 4B). No reduction in necrosis was observed after injection of endoglin-siRNA ECFCs and MSCs (*p<0.0001 vs control-siRNA ECFCs and MSCs). We also found a strong increase in microvessel density after co-injection of control-siRNA ECFCs and MSCs but not with endoglin-siRNA ECFCs and MSCs $\left({ }^{*} \mathrm{p}=0.0002\right.$ vs control-siRNA ECFCs and MSCs; and $\mathrm{p}=0.94$ vs PBS; Figure 4B). Incorporation of human cells into the mouse leg was demonstrated by hCD31 immunohistochemistry (Figure 4A and 4D) and by in situ hybridization of the human ALU sequence (Figure 4A). As expected, we found an increase in human vessel numbers after injection of ECFCs control-siRNA+MSCs but not with endoglin-siRNA ECFCs and MSCs (*p=0.0003 vs ECFCs control-siRNA+MSCs; Figure 4D). Similar results were obtained with in situ hybridization of the human ALU sequence (Figure 4A, central panel). Myofibers (central nuclei), indicative of recovery from hind limb ischemia, were more abundant after ECFCs control-siRNA injection than after PBS or endoglinsiRNA ECFCs injection. Thus, endoglin inhibition in ECFCs strongly reduced muscle revascularization and endothelial cell incorporation in muscle after co-injection with MSCs.

\section{Endoglin silencing in ECFCs does not affect MSC differentiation but regulates adhesion}


ECFCs have been described to induce MSC differentiation into perivascular cells. Because endoglin silencing in ECFCs abolished the beneficial effect of MSC co-injection, we explored the ability of MSCs to acquire the smooth muscle cell (SMC)/pericyte phenotype after co-culture with control-siRNA and endoglin-siRNA ECFCs. As shown in Figure 5, we quantified aSMA, calponin, PDGF $\beta$ and NG2 (Figure 5 A,B,C,D respectively) after coculture for 7 days. We found no difference in MSC perivascular differentiation between control-siRNA and endoglin-siRNA ECFCs. We also explored whether endoglin silencing in ECFCs interfered with a common route of MSC differentiation into adipocytes/osteocytes (Figure 5E,F). No change in differentiation potential was observed whatever the medium tested (Supplementary Figure 6 A,B). We then explored the ability of control-siRNA and endoglin-siRNA ECFCs to interact with MSCs in an adhesion assay. As shown in Figure 6A and 6B, endoglin inhibition in ECFCs significantly reduced ECFC adhesion to MSCs, in both the presence and absence of VEGF $\left({ }^{*} \mathrm{p}=0.002\right)$. 


\section{DISCUSSION}

We show that co-injection of ECFCs and human bone marrow-derived MSCs enhances new blood vessel formation in a mouse model of hind limb ischemia. Indeed, co-injection of MSCs with ECFCs led to a rapid increase in reperfusion by comparison with ECFCs alone. The MSCmediated improvement in ECFC-induced vasculogenesis was driven by ECFC endoglin expression. ECFC endoglin knock-down did not affect MSC differentiation into perivascular cells or another mesenchymal lineage, but it significantly reduced ECFC adhesion to MSCs.

We have previously demonstrated that, in patients with CLI, total BM-MNCs promote neovessel formation $(4,5)$. Co-injection of ECFCs with circulating angiogenic cells (early-EPC) has also been tested in preclinical models of hindlimb ischemia (27). In the present study, we show that MSCs improve ECFC efficiency. There are hundreds of ongoing MSC-based clinical trials targeting a plethora of conditions, including cardiovascular diseases and immune-mediated disorders. The expansion of allogenic and/or autologous MSCs for these trials is well standardized and have clinical grade. Lasala and colleagues $(28,29)$ proposed that combination cell therapy including BM-MNCs and MSCs (a source of perivascular progenitors and angiogenic regulators) might represent a preferential stimulus for the development of blood vessels and for the treatment of medically refractory coronary ischemia (30). MSCs have been described as a good cell therapy product to increase muscle recovery in CLI (2) and we have previously demonstrated that MSCs isolated from CLI patients are functional in a model of HLI (31). In this context, MSCs act as paracrine cells, as no endothelial differentiation is observed (31). The interaction between ECFCs-MSCs has been proposed in the past. However, the efficacy of this combination has only been described in extracellular matrices (ECM, Matrigel or collagen implants) $(9,26)$ implanted in immunodeficient mice. In these models, MSCs are thought to 
support vasculogenic processes by their ability to transdifferentiate into perivascular cells when they interact with endothelial cells or endothelial progenitors $(9,32,33)$. A paracrine effect of MSCs on ECFC vasculogenic potential has been described in ECM models (34). Conversely, ECFCs can also influence MSC maturation. In a very elegant study, Melero-Martin et al. showed that ECFCs can function as paracrine mediators before the establishment of perfusion (35), modulating the regenerative potential of MSCs. They also showed that ECFC trophic support of MSCs was driven by PDGF-BB/PDGFR- $\beta$ signaling. Thus, ECFCs and MSCs are real partners in vasculogenesis. In line with these results, we found that MSCs enhanced the capacity of ECFCs to accelerate recovery after hind limb ischemia. As demonstrated here, after 7 days, ECFC+MSC coinjection was more efficient than ECFC injection alone, with better blood flow, better muscle recovery, and less necrosis. MSCs enhanced ECFC engraftment after adhesion, resulting in improved regenerative potential. To our knowledge, no data have been published on the coinjection of ECFCs + MSCs in hind limb ischemia or CLI, and no mechanism has been proposed to explain cooperation between these cells. We demonstrate here that MSC regenerative support is driven by adhesion to the ECFC membrane via endoglin. This endoglin-mediated adhesion enabled early engraftment of transplanted ECFCs. Interactions between endothelial cells and MSCs are crucial for vascular development, and go from perivascular recruitment by PDGF-BB secretion to vessel stabilization mediated, at least in part, by angiopoietin-Tie2 pathways (36). It has been also reported that MSC-to-perivascular cells differentiation is driven by MSC interaction with endothelial cells by a Jagged/Notch dependent mechanism. These results are supported by mutations in Jagged1, mutations in Notch1, Notch1/Notch4 or components of the Notch signaling cascade that result in mouse embryo lethality associated with severe vascular defects; zebrafish genetic studies have also demonstrated Notch functions in arterial/venous specification (37). Thus, cooperation between the two cell types allows perivascular cell 
formation and stable vessel formation. We demonstrate here that endoglin does not modify this MSC perivascular differentiation. Human endothelial cells and ECFCs express endoglin, alias CD105, on their membrane, with a critical physiological role in the cardiovascular system (38). Endoglin is an auxiliary receptor for the transforming growth factor $\beta$ (TGF- $\beta$ ) family of proteins that is essential for angiogenesis (39). Indeed, endoglin knockdown (Endoglin ${ }^{-/}$) mouse embryos die because of vascular and cardiac abnormalities (40). Furthermore, mutations in the human endoglin gene that lead to endoglin haploinsufficiency are responsible for hereditary hemorrhagic telangiectasia (HHT) type 1 (41). Endoglin, recently defined as an adhesion molecule $(11,12)$, seems to participate in ECFC-MSC cooperation and to allow early ECFC engraftment in mouse muscle in the hind limb ischemia model. As we have already demonstrated, endothelial endoglin interacts with mural cells during vessel stabilization, via its RGD motif that can be recognized by MSC integrins (11). In the present study, endoglin inhibition seemed to compromise adhesion between ECFCs and MSCs in the hind limb ischemia model. This abrogated the enhanced engraftment and neovessel regeneration.

Our study has some limitations. First, endoglin is expressed by all vessels, from embryonic (42) to adult life. The endoglin gene sequence is well conserved and shows strong homology across species (43). Thus, in the normal hind limb, endoglin is expressed by all endothelial cells, in humans as in mice. Endoglin expression has been reported to increase in active endothelium undergoing angiogenesis during cancer (44), chronic colitis (45), myocardial infarction (46) and vascular injury (47). Thus, we cannot exclude an effect of mouse endoglin on muscle recovery. However, our study focused on endoglin expression by human ECFC from a cell-therapy point of view, and on the possible benefits of ECFC-MSC co-injection. Endoglin silencing in ECFC affects their ability to interact with MSC and, consequently, cell engraftment and muscle 
regeneration. Second, we cannot exclude the possibility that TGF- $\beta$ family members secreted by MSCs are involved in MSC-ECFC cross-talk. However, our results support a critical role of endoglin in adhesion between MSCs and ECFCs, a function that we have previously shown to be independent of the TGF- $\beta$ system during inflammation (12). On the other hand, MSCs secrete multiple TGF- $\beta$ and BMP members (48). We have previously reported that TGF- $\beta$ and BMP2/4 induce ECFC to adopt a proangiogenic phenotype $(5,49)$. When secreted by MSCs, this ligand could modify ECFC endoglin expression. Thus, we cannot rule out TGF- $\beta$ family member participation in MSC/ECFC cross-talk.

In conclusion, our data support the use of MSCs to improve therapeutic ECFC engraftment in hind limb ischemia. We demonstrate that MSC-ECFC coinjection accelerates muscle recovery through an endoglin-dependent mechanism. Endoglin from endothelial progenitors is involved in MSC-ECFC adhesion, and endoglin inhibition abolishes MSCs' ability to support blood vessel formation in vivo. A better understanding of this endoglin pathway in vasculogenic cells could lead to new revascularization strategies. 


\section{ACKNOWLEDGMENTS}

This work was supported by grants from Région Ile de France-CORDDIM (Domaine d'intérêt majeur Cardiovasculaire Obésité Rein Diabète) and the Conny-Maeva Charitable Foundation. Carmelo Bernabeu is supported by Ministerio de Economia y Competitividad of Spain (SAF2013-43421-R to C.B.).

Elisa Rossi's salary is supported by a grant from the Conny-Maeva Charitable Foundation.

Nour Bacha and Celine Goyard's salaries are supported by a grant from Fond de Dotation pour la Recherche en Santé Respiratoire.

\section{Conflict-of-interest disclosure}

The authors declare no competing financial interests

\section{Authorship}

Contributions: E.R. and D.M.S. designed the research, analyzed the data, and wrote the paper; E.R., C.G, N.B, N.G. and B.D. performed in vivo assays; E.R, N.G. and C.G performed the in vitro assays and cell culture; E.R. and V.M performed immunohistochemistry. E.R. performed immunofluorescence. A.C. performed MSC extraction and differentiation. C.B and O.S. provided helpful suggestions and contributed to writing the manuscript; D.M.S. supervised the research, wrote the paper and provided funding support. 


\section{Figure legends}

Figure 1. Injection of ECFCs and MSCs in nude mice after hind limb ischemia.

A. Nude mice (n=6 PBS, $n=6$ ECFCs, $n=6$ ECFC+MSC) underwent Doppler analysis to determine cutaneous blood flow on days 1, 7 and 14 .

B. Ratio between the ischemic/non ischemic hind limbs on days 1, 7 and 14 .

Figure 2. Histomorphologic analysis of ischemic muscle 14 days after ECFC and MSC injection

A. H\&E of frozen left leg sections. Asterisks (*) indicate myocyte necrosis, white arrows indicate myofibers with central nuclei, present only in the left leg after ECFCs and ECFC+MSC injection. Immunohistochemistry for mouse CD31 (mCD31) and human CD31 (hCD31) are shown (20 and 40X magnification for human CD31). In situ hybridization for the human ALU sequence is shown in central panel and display human nuclei by the probe against Alu repeats (purple, arrows). 20X magnification: scale bar $100 \mu \mathrm{m}, 40 \mathrm{X}$ magnification :scale bar $50 \mu \mathrm{m}$

B. Necrosis (grade 1, Willet criteria), higher in PBS samples.

C. Immunohistochemistry for mCD31 indicating capillary density.

D. Human CD31 detects ECFC incorporation. Quantification realized at 20X magnification. 
Figure 3. Effect of ECFC endoglin inhibition on the efficacy of ECFC/MSC co-injection in nude mice with hind limb ischemia.

A. Nude mice ( $n=6$ PBS, $n=6$ ECFCs control-siRNA+MSCs, $n=6$ ECFCs endoglinsiRNA+MSCs) underwent Doppler analysis of cutaneous blood flow on days 1, 7 and 14 .

B. Ratio between ischemic/non ischemic legs on days 1, 7 and 14 .

Figure 4. Histomorphologic analysis of ischemic muscle 14 days after injection of ECFCs (silenced or not for endoglin) and MSCs

A. H\&E of right and left legs on frozen tissue. Asterisks $\left(^{*}\right)$ indicate myocyte necrosis. Necrosis was present in the PBS and ECFC endoglin-siRNA +MSC conditions. Arrows: myofibers with central nuclei, present in the left leg after ECFC endoglin-siRNA + MSC injection. Immunohistochemistry for mCD31 and hCD31 are shown (20 and 40X magnification for human CD31). In situ hybridization of the human ALU sequence in the central panels display human nuclei by the probe against Alu repeats (purple, arrows). 20X magnification: scale bar $100 \mu \mathrm{m}$, 40X magnification :scale bar $50 \mu \mathrm{m}$

B. Necrosis. Grade 1 necrosis $(<50 \%$, Willet's criteria) was present in PBS and ECFC endoglinsiRNA conditions.

C. mCD31 reveals higher capillary density in ECFC control-siRNA conditions than in ECFC endoglin-siRNA conditions.

D. hCD31 reveals ECFC incorporation. Quantification realized at 20X magnification. c-siRNA, control-siRNA; e-siRNA, endoglin-siRNA. 
Figure 5. MSC transdifferentiation analyzed by immunofluorescence after ECFC+MSC coculture with or without ECFC endoglin silencing. No difference has been found between ECFCs control-siRNA and ECFCs endoglin-siRNA.

A. Coculture of MSCs labelled by aSMA (green) and ECFCs by vWF (red). 40X magnification: scale bar $50 \mu \mathrm{m}$

B. Coculture of MSCs labelled by Calponin and ECFCs by vWF. 40X magnification: scale bar $50 \mu \mathrm{m}$

C. Coculture of MSCs labelled by PDGF $\beta$ (green) and ECFCs by vWF. 40X magnification: scale bar $50 \mu \mathrm{m}$

D. Coculture of MSCs labelled by NG2 (green) and ECFCs by vWF. 40X magnification: scale bar $50 \mu \mathrm{m}$

E. ECFCs endoglin-siRNA +MSCs and F. ECFCs control-siRNA +MSCs after 7 days of coculture in EGM2 medium and 2 weeks of treatment with adipogenic/osteogenic differentiation media. Stains for adipogenic (Nile Red, NR) and osteogenic (alkaline phosphatase, ALP / Alizarine Red, AR) differentiation were used. 10X magnification: scale bar $200 \mu \mathrm{m}$

\section{Figure 6. In vitro ECFC adhesion to an MSC monolayer}

A. Adhesion of ECFCs labelled with CFSE to an MSC monolayer. Endoglin inhibition reduced

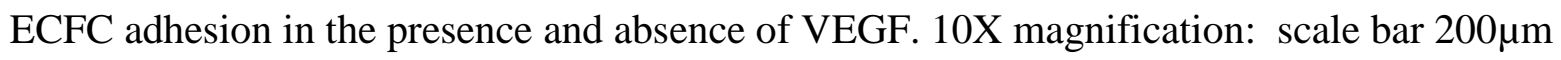

B. ECFC adhesion to an MSC monolayer. 


\section{$\underline{\text { References }}$}

1. Powell RJ. Update on clinical trials evaluating the effect of biologic therapy in patients with critical limb ischemia. J Vasc Surg. 2012; 56: 264-6.

2. Silvestre JS, Smadja DM, Levy BI. Postischemic revascularization: from cellular and molecular mechanisms to clinical applications. Physiol Rev. 2013; 93: 1743-802.

3. Tateishi-Yuyama E, Matsubara H, Murohara $\mathrm{T}$, et al. Therapeutic angiogenesis for patients with limb ischaemia by autologous transplantation of bone-marrow cells: a pilot study and a randomised controlled trial. Lancet. 2002; 360: 427-35.

4. Duong-Van-Huyen JP, Smadja DM, Bruneval P, et al. Bone marrow-derived mononuclear cell therapy induces distal angiogenesis after local injection in critical leg ischemia. Mod Pathol. 2008; 21: 837-46.

5. Smadja DM, Bieche I, Silvestre JS, et al. Bone morphogenetic proteins 2 and 4 are selectively expressed by late outgrowth endothelial progenitor cells and promote neoangiogenesis. Arterioscler Thromb Vasc Biol. 2008; 28: 2137-43.

6. Pignon B, Sevestre MA, Chatelain D, et al. Histological changes after implantation of autologous bone marrow mononuclear cells for chronic critical limb ischemia. Bone Marrow Transplant. 2007; 39: 647-8.

7. Yoder MC, Mead LE, Prater D, et al. Redefining endothelial progenitor cells via clonal analysis and hematopoietic stem/progenitor cell principals. Blood. 2007; 109: 1801-9.

8. Smadja DM, Duong-van-Huyen JP, Dal Cortivo L, et al. Early endothelial progenitor cells in bone marrow are a biomarker of cell therapy success in patients with critical limb ischemia. Cytotherapy. 2012; 14: 232-9.

9. Melero-Martin JM, De Obaldia ME, Kang SY, et al. Engineering robust and functional vascular networks in vivo with human adult and cord blood-derived progenitor cells. Circ Res. 2008; 103: 194-202.

10. Rossi E, Lopez-Novoa JM, Bernabeu C. Endoglin involvement in integrin-mediated cell adhesion as a putative pathogenic mechanism in hereditary hemorrhagic telangiectasia type 1 (HHT1). Front Genet. 2015; 5: 457.

11. Rossi E, Smadja DM, Boscolo E, et al. Endoglin regulates mural cell adhesion in the circulatory system. Cell Mol Life Sci. 2016; 73: 1715-39.

12. Rossi E, Sanz-Rodriguez F, Eleno N, et al. Endothelial endoglin is involved in inflammation: role in leukocyte adhesion and transmigration. Blood. 2013; 121: 403-15.

13. Freida $D$, Lecourt $S$, Cras $A$, et al. Human bone marrow mesenchymal stem cells regulate biased DNA segregation in response to cell adhesion asymmetry. Cell Rep. 2013; 5: 601-10.

14. Dominici M, Le Blanc K, Mueller I, et al. Minimal criteria for defining multipotent mesenchymal stromal cells. The International Society for Cellular Therapy position statement. Cytotherapy. 2006; 8: 315-7.

15. Cochain C, Rodero MP, Vilar J, et al. Regulation of monocyte subset systemic levels by distinct chemokine receptors controls post-ischaemic neovascularization. Cardiovasc Res. 2010; 88: 186-95.

16. HoWangYin KY, Loinard C, Bakker W, et al. HIF-prolyl hydroxylase 2 inhibition enhances the efficiency of mesenchymal stem cell-based therapies for the treatment of critical limb ischemia. Stem Cells. 2014; 32: 231-43.

17. Smadja DM, Guerin $\mathrm{CL}$, Boscolo $\mathrm{E}$, et al. alpha6-Integrin is required for the adhesion and vasculogenic potential of hemangioma stem cells. Stem Cells. 2014; 32: 684-93.

18. Swirski FK, Berger CR, Figueiredo JL, et al. A near-infrared cell tracker reagent for multiscopic in vivo imaging and quantification of leukocyte immune responses. PLoS One. 2007; 2: e1075. 
19. Willett $C G$, Schiller AL, Suit HD, et al. The histologic response of soft tissue sarcoma to radiation therapy. Cancer. 1987; 60: 1500-4.

20. Zheng JK, Wang Y, Karandikar A, et al. Skeletal myogenesis by human embryonic stem cells. Cell Res. 2006; 16: 713-22.

21. Paoni NF, Peale F, Wang F, et al. Time course of skeletal muscle repair and gene expression following acute hind limb ischemia in mice. Physiol Genomics. 2002; 11: 263-72.

22. Heeschen C, Lehmann R, Honold J, et al. Profoundly reduced neovascularization capacity of bone marrow mononuclear cells derived from patients with chronic ischemic heart disease. Circulation. 2004; 109: 1615-22.

23. Hill JM, Zalos G, Halcox JP, et al. Circulating endothelial progenitor cells, vascular function, and cardiovascular risk. N Engl J Med. 2003; 348: 593-600.

24. Smadja DM, Bieche I, Emmerich J, et al. PAR-1 activation has different effects on the angiogenic activity of endothelial progenitor cells derived from human adult and cord blood. J Thromb Haemost. 2006; 4: 2729-31.

25. Smadja DM, Mauge L, Susen S, et al. Blood outgrowth endothelial cells from cord blood and peripheral blood: angiogenesis-related characteristics in vitro: a rebuttal. J Thromb Haemost. 2009; 7: 504-6; author reply 6-8.

26. Au P, Daheron LM, Duda DG, et al. Differential in vivo potential of endothelial progenitor cells from human umbilical cord blood and adult peripheral blood to form functional long-lasting vessels. Blood. 2008; 111: 1302-5.

27. Yoon $\mathrm{CH}$, Hur J, Park KW, et al. Synergistic neovascularization by mixed transplantation of early endothelial progenitor cells and late outgrowth endothelial cells: the role of angiogenic cytokines and matrix metalloproteinases. Circulation. 2005; 112: 1618-27.

28. Lasala GP, Silva JA, Gardner PA, et al. Combination stem cell therapy for the treatment of severe limb ischemia: safety and efficacy analysis. Angiology. 2010; 61: 551-6.

29. Lasala GP, Silva JA, Minguell JJ. Therapeutic angiogenesis in patients with severe limb ischemia by transplantation of a combination stem cell product. J Thorac Cardiovasc Surg. 2011;

30. Lasala GP, Silva JA, Kusnick BA, et al. Combination stem cell therapy for the treatment of medically refractory coronary ischemia: a Phase I study. Cardiovasc Revasc Med. 2011; 12: 29-34.

31. Smadja DM, d'Audigier C, Guerin CL, et al. Angiogenic potential of BM MSCs derived from patients with critical leg ischemia. Bone Marrow Transplant. 2012; 47: 997-1000.

32. Melero-Martin JM, Khan ZA, Picard A, et al. In vivo vasculogenic potential of human bloodderived endothelial progenitor cells. Blood. 2007; 109: 4761-8.

33. Au P, Tam J, Fukumura $D$, et al. Bone marrow-derived mesenchymal stem cells facilitate engineering of long-lasting functional vasculature. Blood. 2008; 111: 4551-8.

34. Smadja DM, Levy M, Huang L, et al. Treprostinil indirectly regulates endothelial colony forming cell angiogenic properties by increasing VEGF-A produced by mesenchymal stem cells. Thromb Haemost. 2015; 114:

35. Lin RZ, Moreno-Luna R, Li D, et al. Human endothelial colony-forming cells serve as trophic mediators for mesenchymal stem cell engraftment via paracrine signaling. Proc Natl Acad Sci U S A. 2014; 111: 10137-42.

36. Winkler EA, Bell RD, Zlokovic BV. Central nervous system pericytes in health and disease. Nat Neurosci. 2011; 14: 1398-405.

37. Shawber CJ, Kitajewski J. Notch function in the vasculature: insights from zebrafish, mouse and man. Bioessays. 2004; 26: 225-34.

38. Lopez-Novoa JM, Bernabeu C. The physiological role of endoglin in the cardiovascular system. Am J Physiol Heart Circ Physiol. 2010; 299: H959-74. 
39. Lastres $\mathrm{P}$, Letamendia $\mathrm{A}$, Zhang $\mathrm{H}$, et al. Endoglin modulates cellular responses to TGF-beta 1. J Cell Biol. 1996; 133: 1109-21.

40. Mahmoud M, Upton PD, Arthur HM. Angiogenesis regulation by TGFbeta signalling: clues from an inherited vascular disease. Biochem Soc Trans. 2011; 39: 1659-66.

41. Shovlin CL. Hereditary haemorrhagic telangiectasia: pathophysiology, diagnosis and treatment. Blood Rev. 2010; 24: 203-19.

42. Roques M, Durand C, Gautier R, et al. Endoglin expression level discriminates long-term hematopoietic from short-term clonogenic progenitor cells in the aorta. Haematologica. 2012; 97: 975-9.

43. Rossi E, Langa C, Gilsanz A, et al. Characterization of chicken endoglin, a member of the zona pellucida family of proteins, and its tissue expression. Gene. 2012; 491: 31-9.

44. Kuiper P, Hawinkels LJ, de Jonge-Muller ES, et al. Angiogenic markers endoglin and vascular endothelial growth factor in gastroenteropancreatic neuroendocrine tumors. World J Gastroenterol. 2011; 17: 219-25.

45. Ardelean DS, Yin $M$, Jerkic $M$, et al. Anti-VEGF therapy reduces intestinal inflammation in Endoglin heterozygous mice subjected to experimental colitis. Angiogenesis. 2014; 17: 641-59.

46. van Laake LW, van den Driesche S, Post S, et al. Endoglin has a crucial role in blood cell-mediated vascular repair. Circulation. 2006; 114: 2288-97.

47. Munoz R, Arias Y, Ferreras JM, et al. Transient injury-dependent up-regulation of CD105 and its specific targeting with an anti-vascular anti-mouse endoglin-nigrin b immunotoxin. Med Chem. 2012; 8: 996-1002.

48. Dexheimer V, Gabler J, Bomans K, et al. Differential expression of TGF-beta superfamily members and role of Smad1/5/9-signalling in chondral versus endochondral chondrocyte differentiation. Sci Rep. 2016; 6: 36655.

49. Evrard SM, d'Audigier C, Mauge L, et al. The profibrotic cytokine transforming growth factorbeta1 increases endothelial progenitor cell angiogenic properties. J Thromb Haemost. 2012; 10: 670-9. 


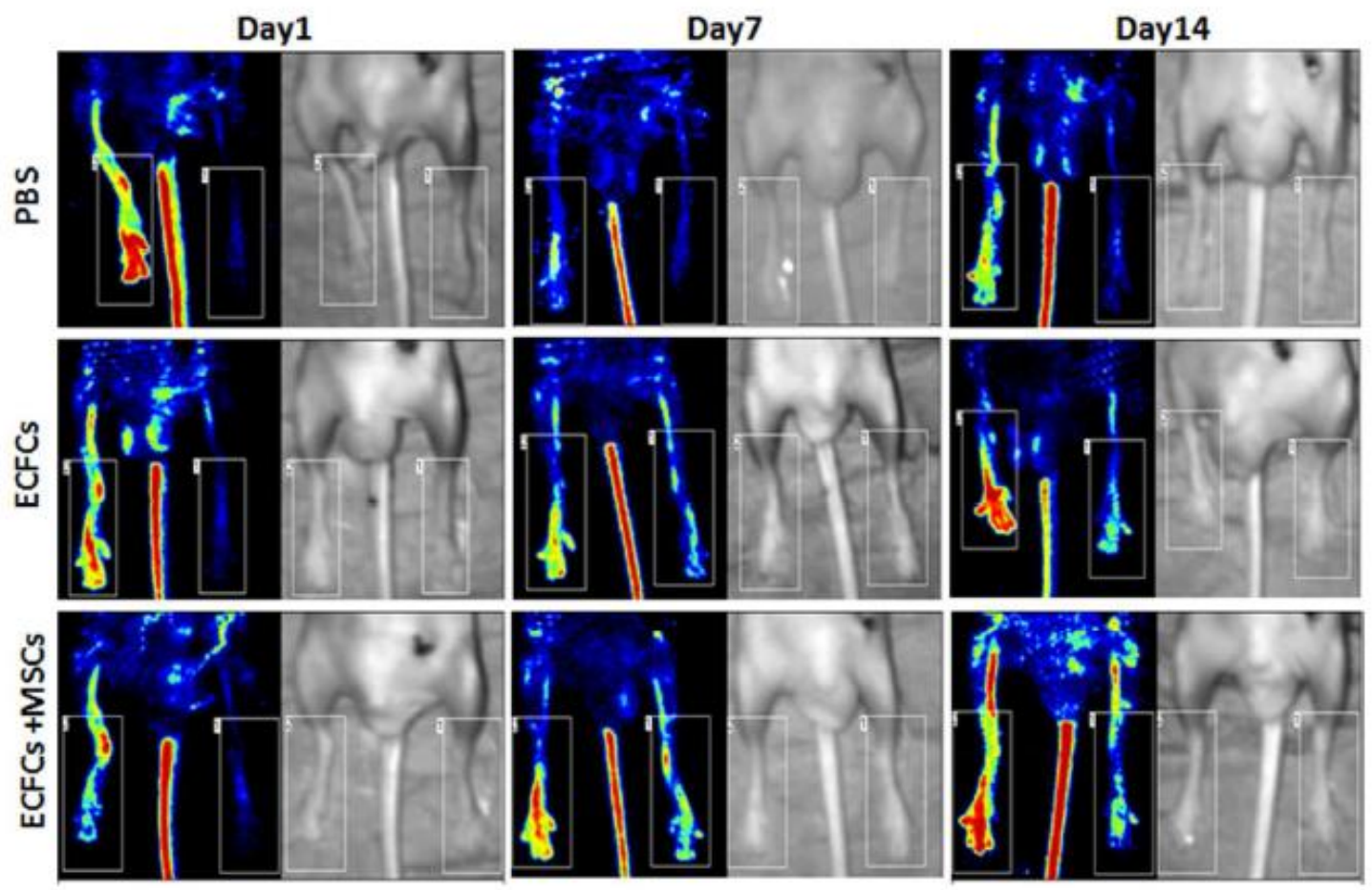

Figure 1A

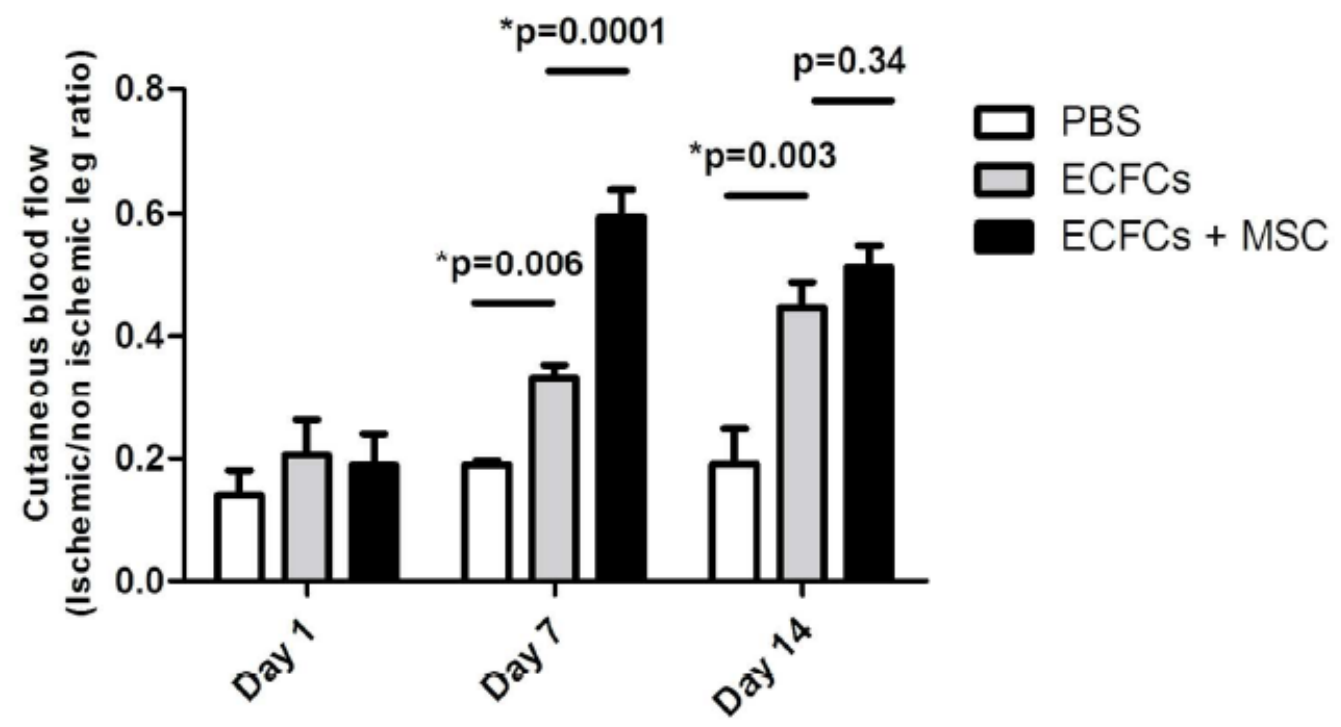

Figure 1B 


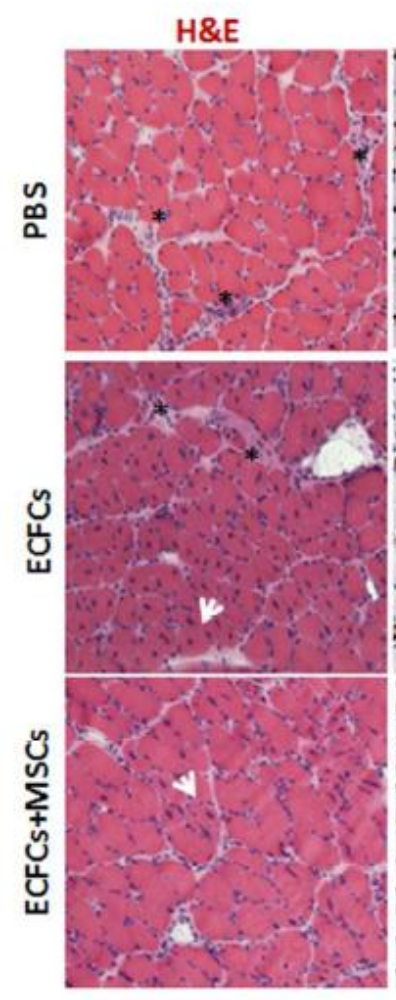

Mouse CD31

Human CD31

Human ALU
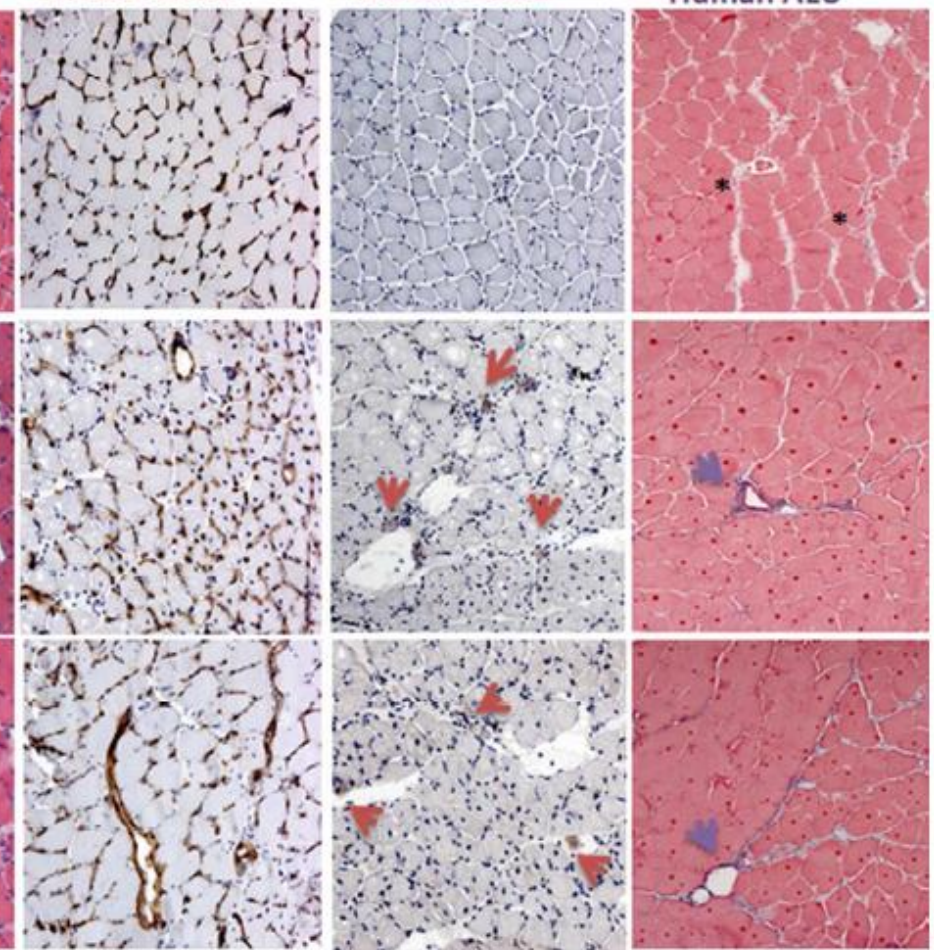

Figure 2A

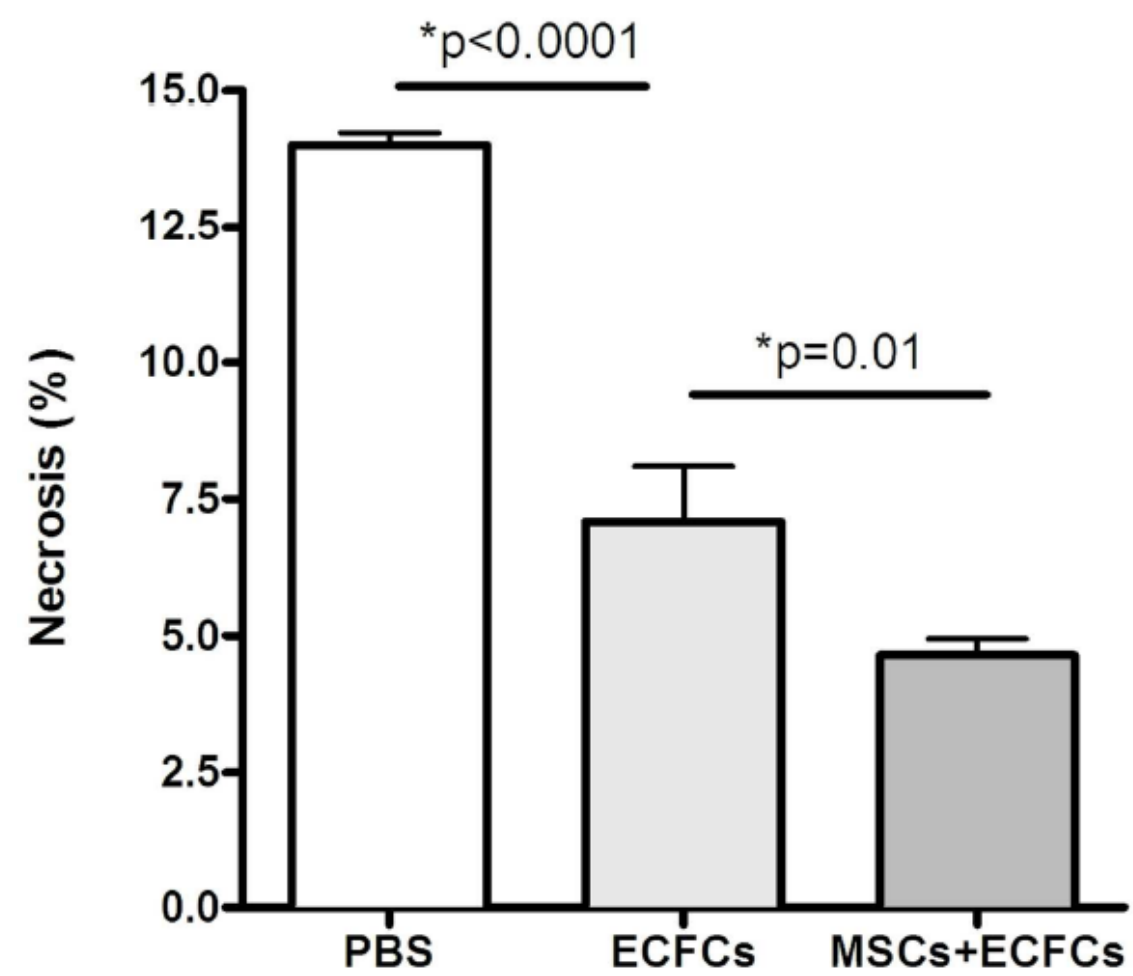

Figure 2B 


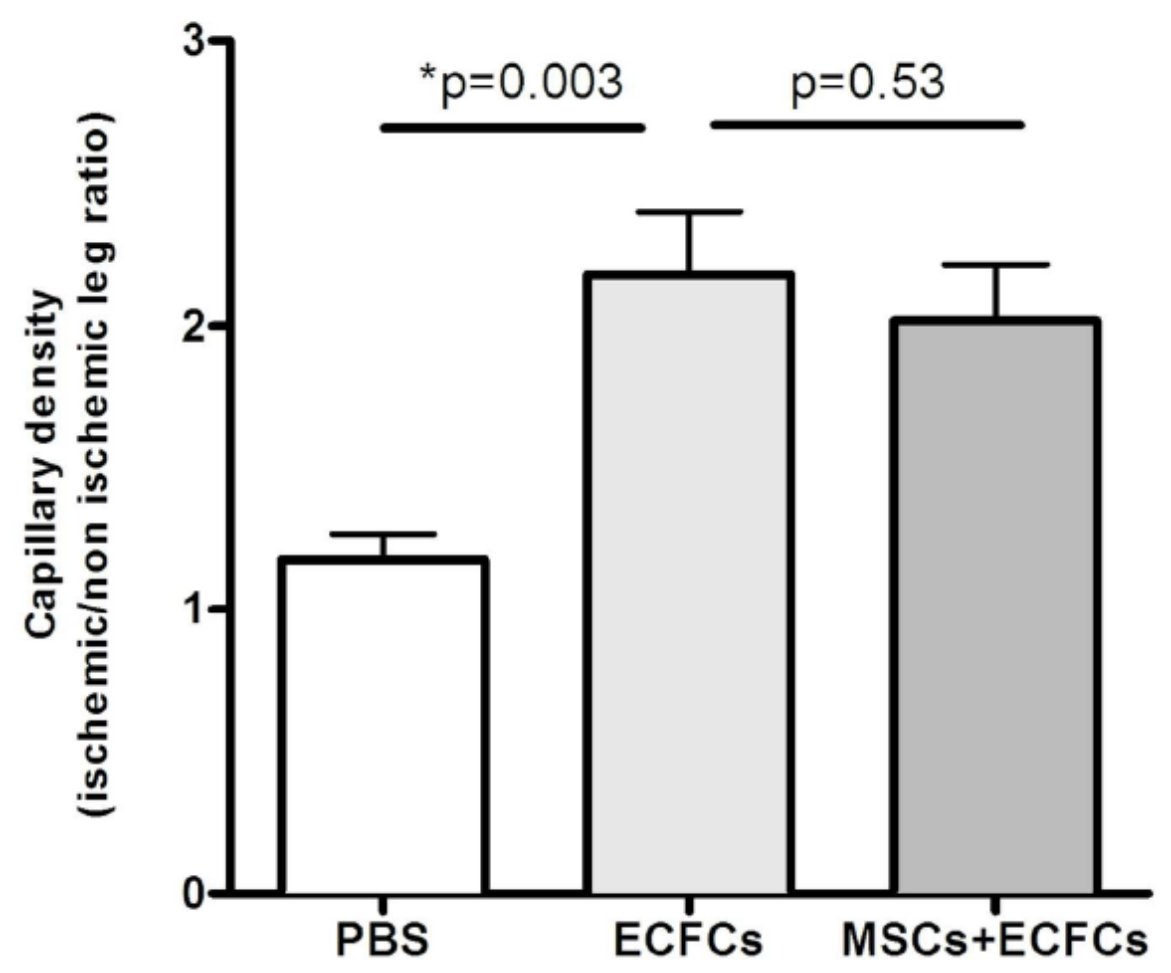

Figure 2C

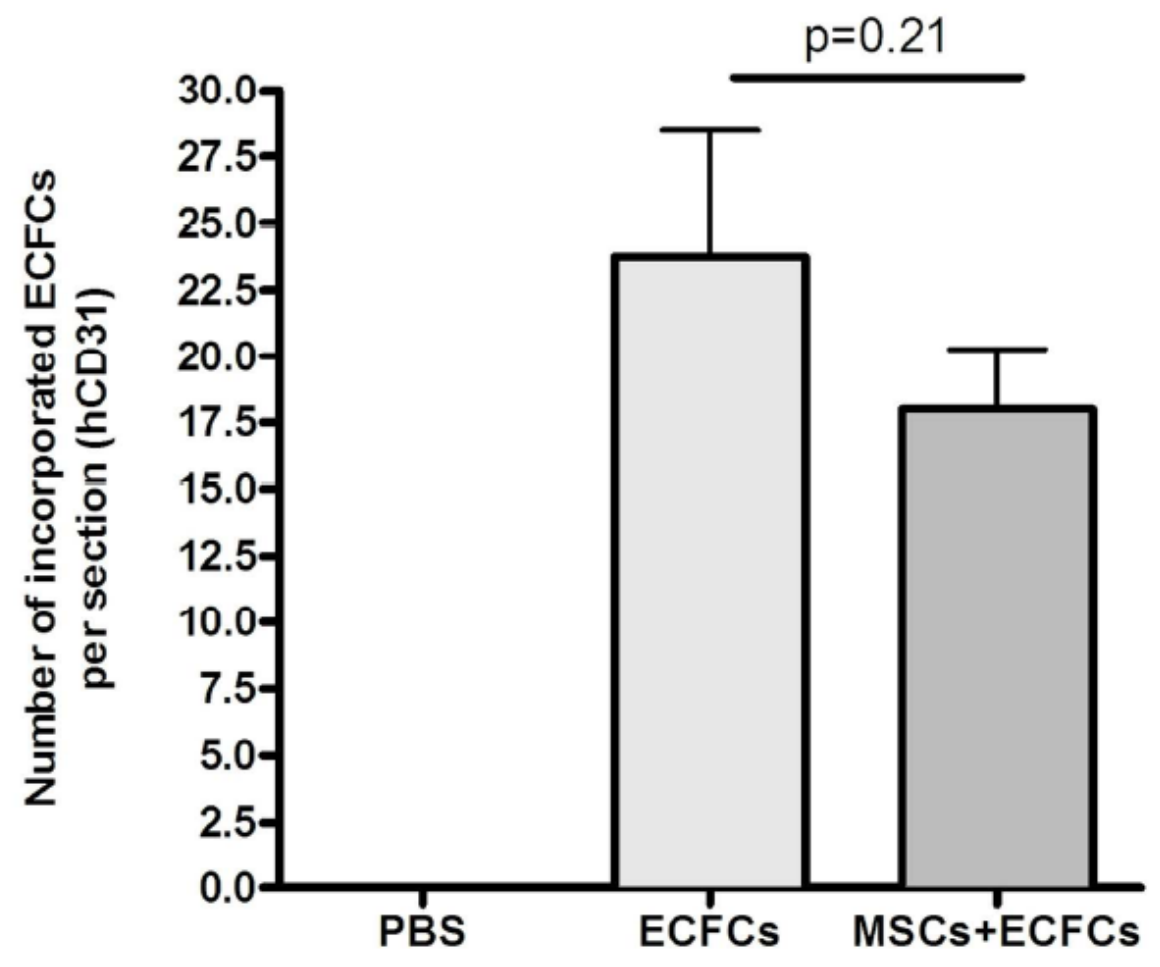

Figure 2D 

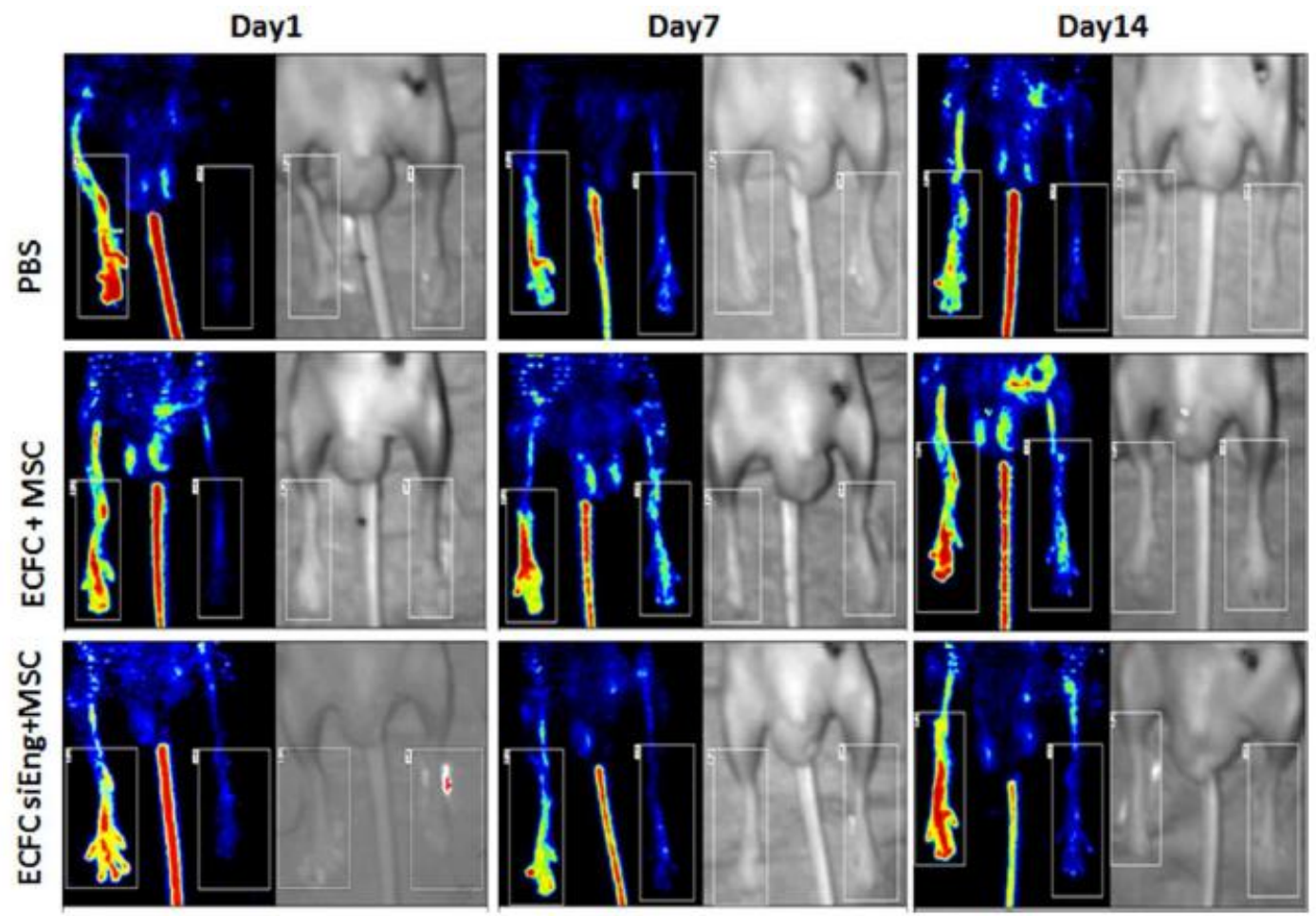

Figure 3A

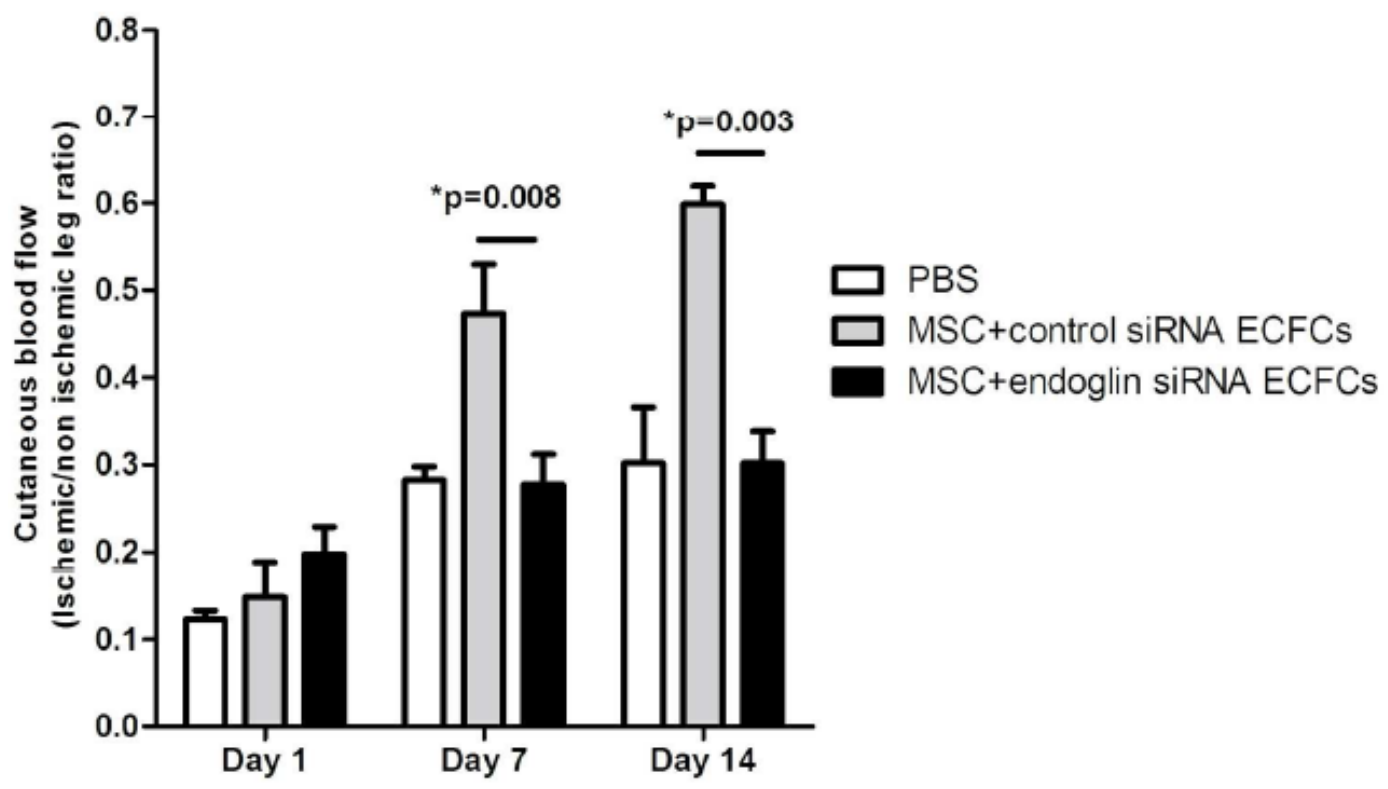

Figure 3B 

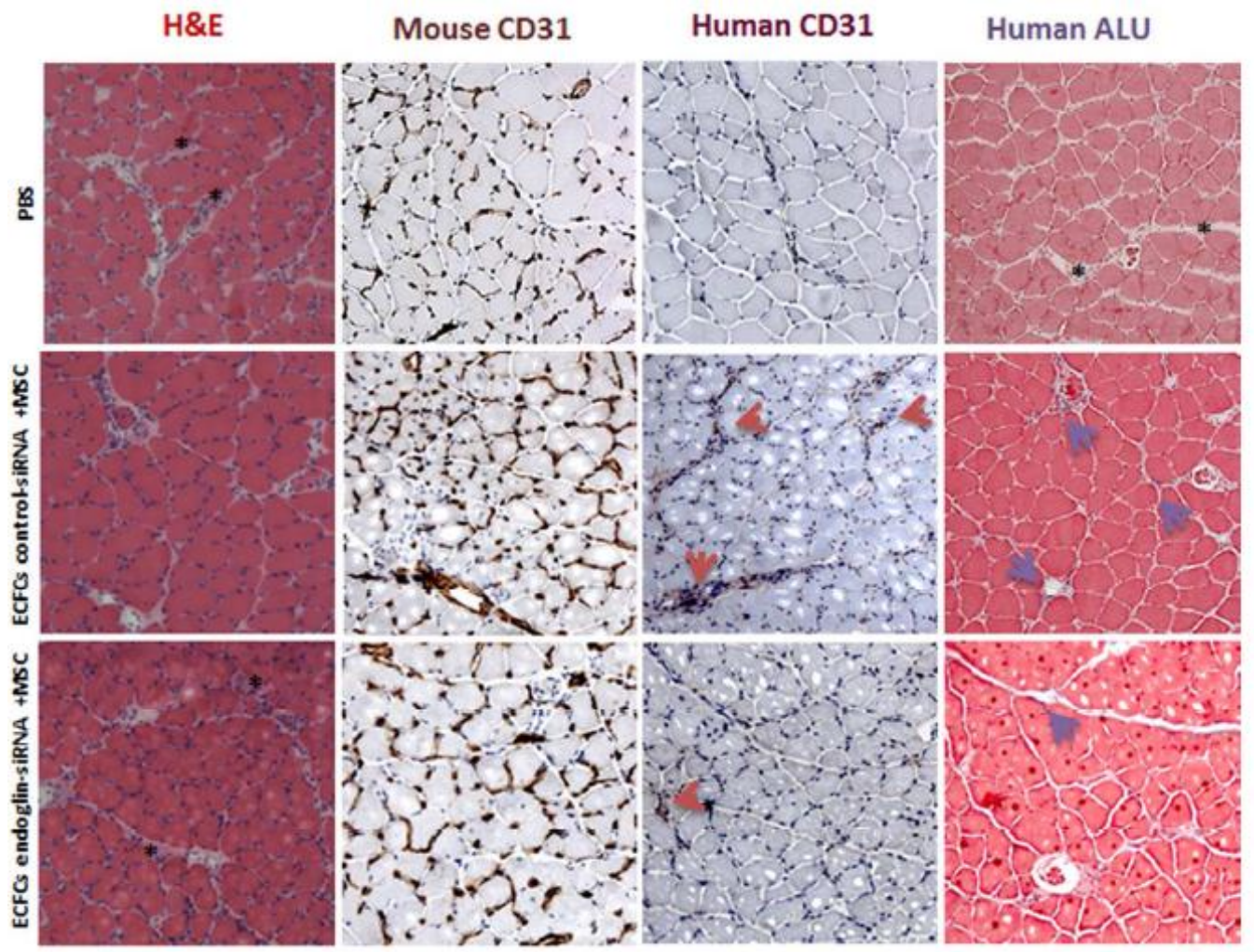

Figure 4A

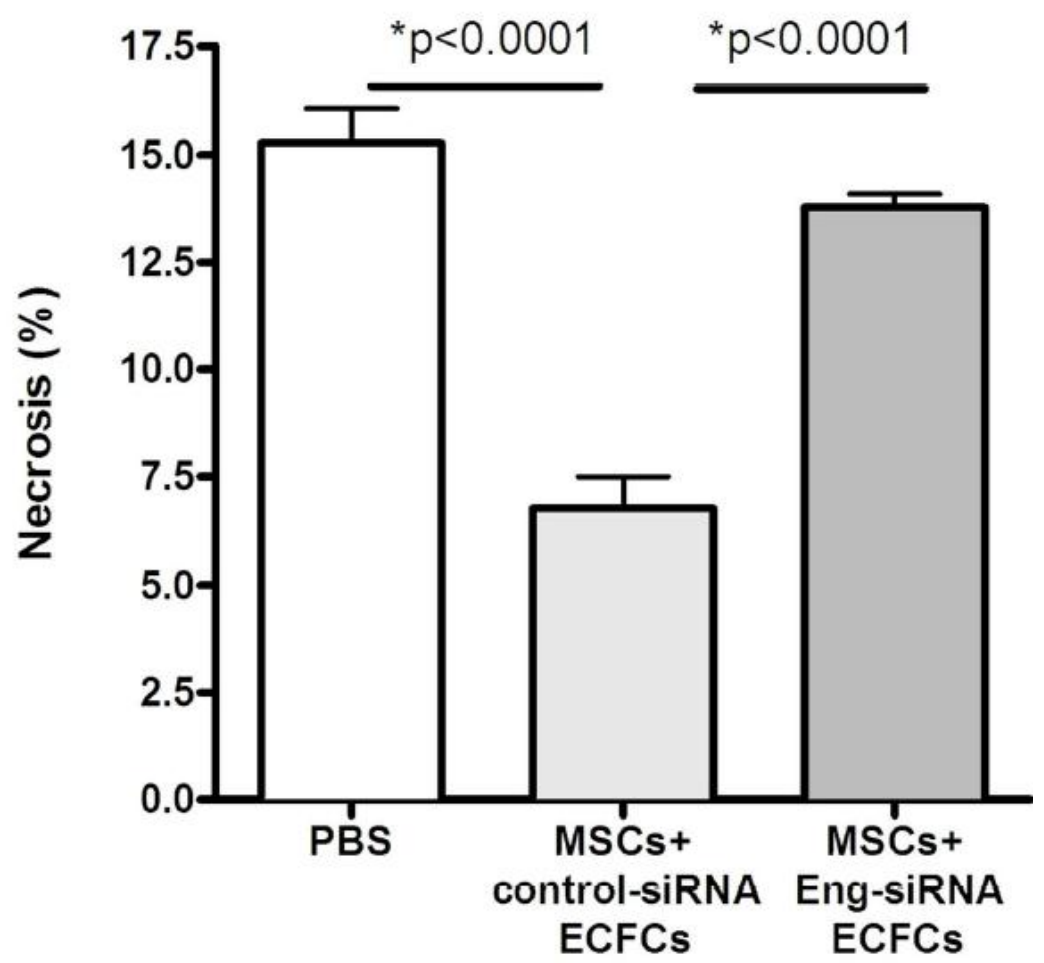

Figure 4B 


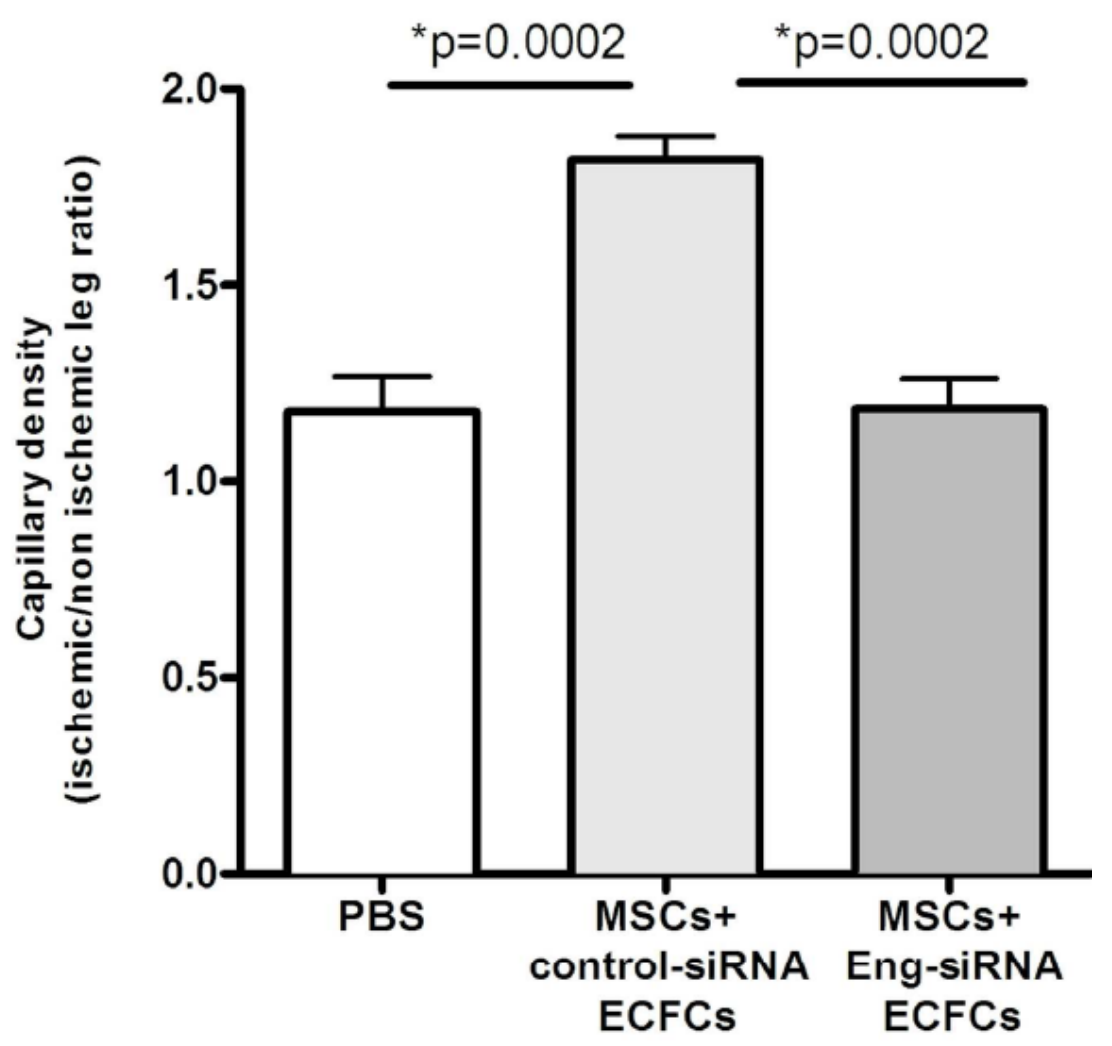

Figure 4C

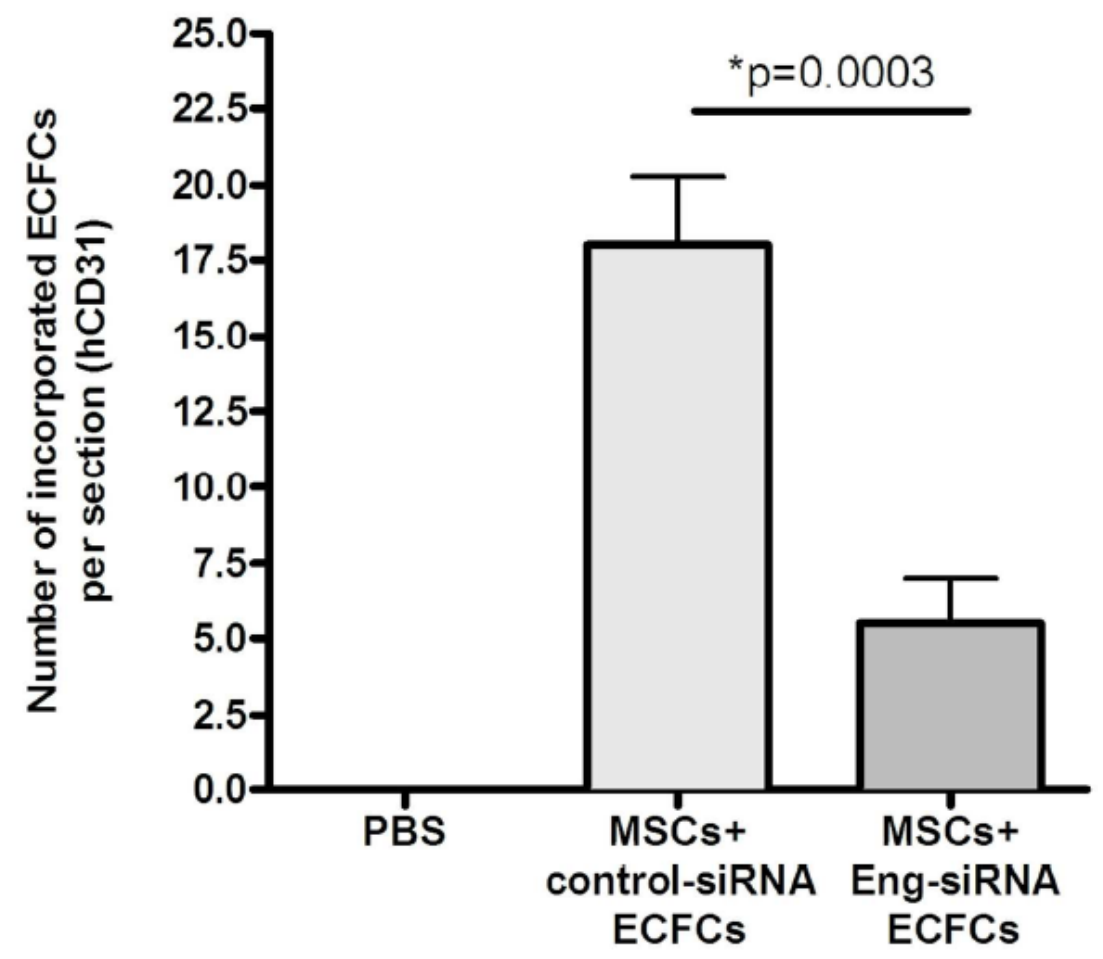

Figure 4D 

ECFCs control-siRNA+MSCs
ECFCs endoglin-siRNA+MSCs
(EGM2)
ECFCs control-siRNA+MSCs ECFCs endoglin-siRNA+MSCs$$
\text { (EGM2) }
$$
(EGM2)
(EGM2)

ECFCvWF nuclei TOPRO ECFCvWF nuclei TOPRO ECFC vWF nuclei TOPRO ECFCvWF nuclei TOPRO
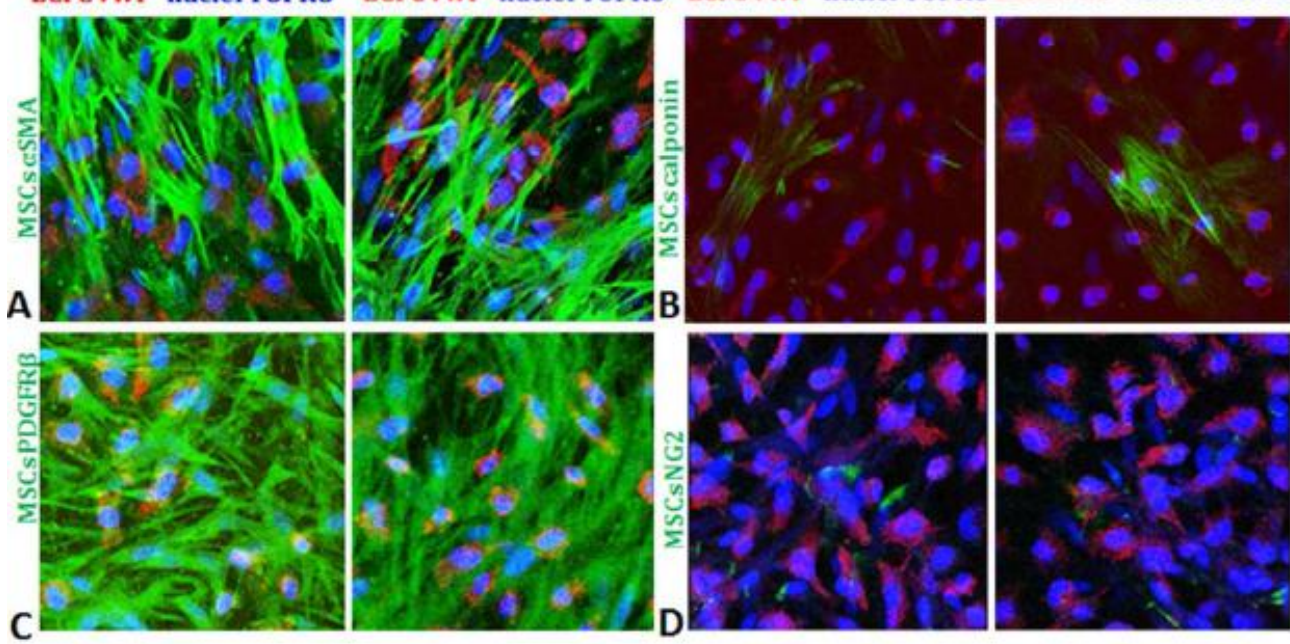

Figure 5 A-D

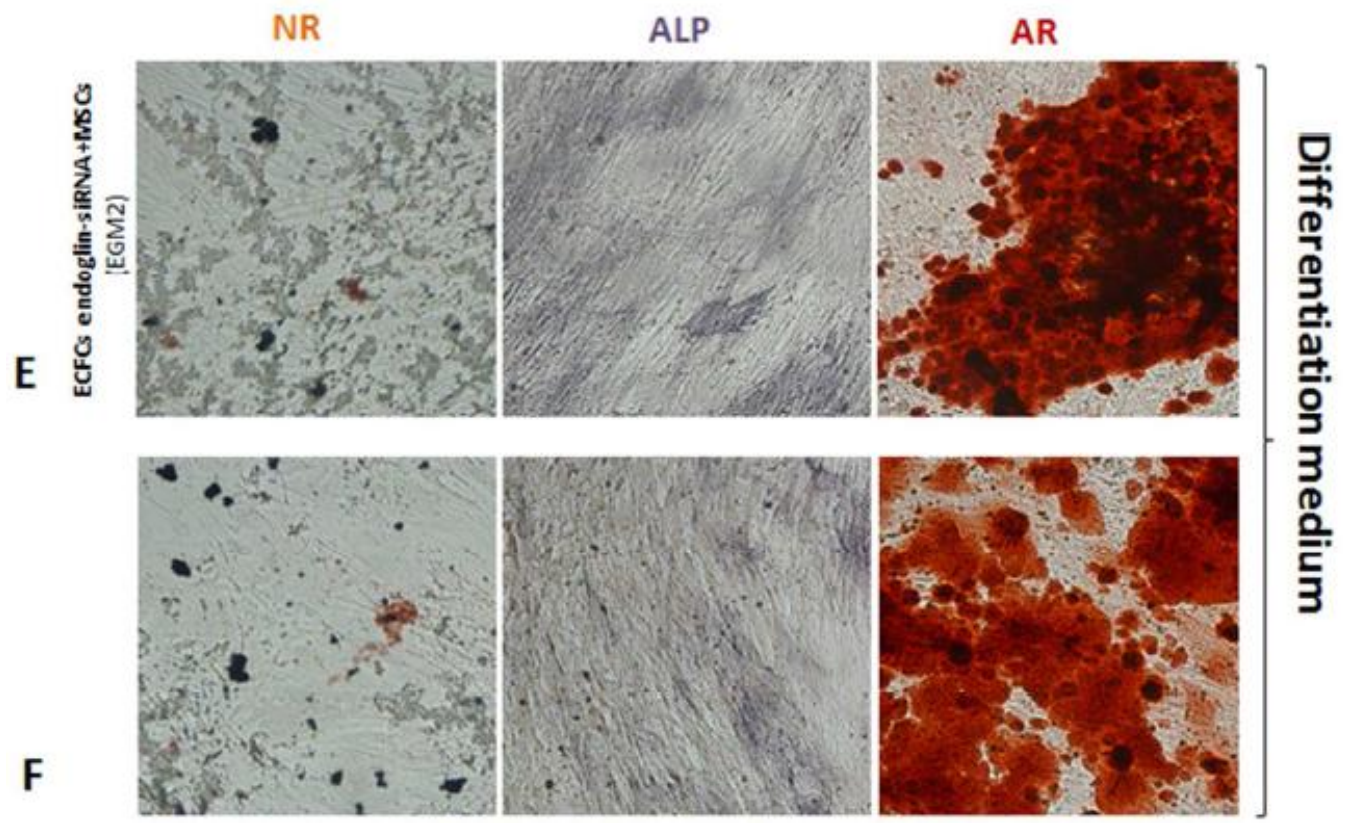

Figure 5 E,F 


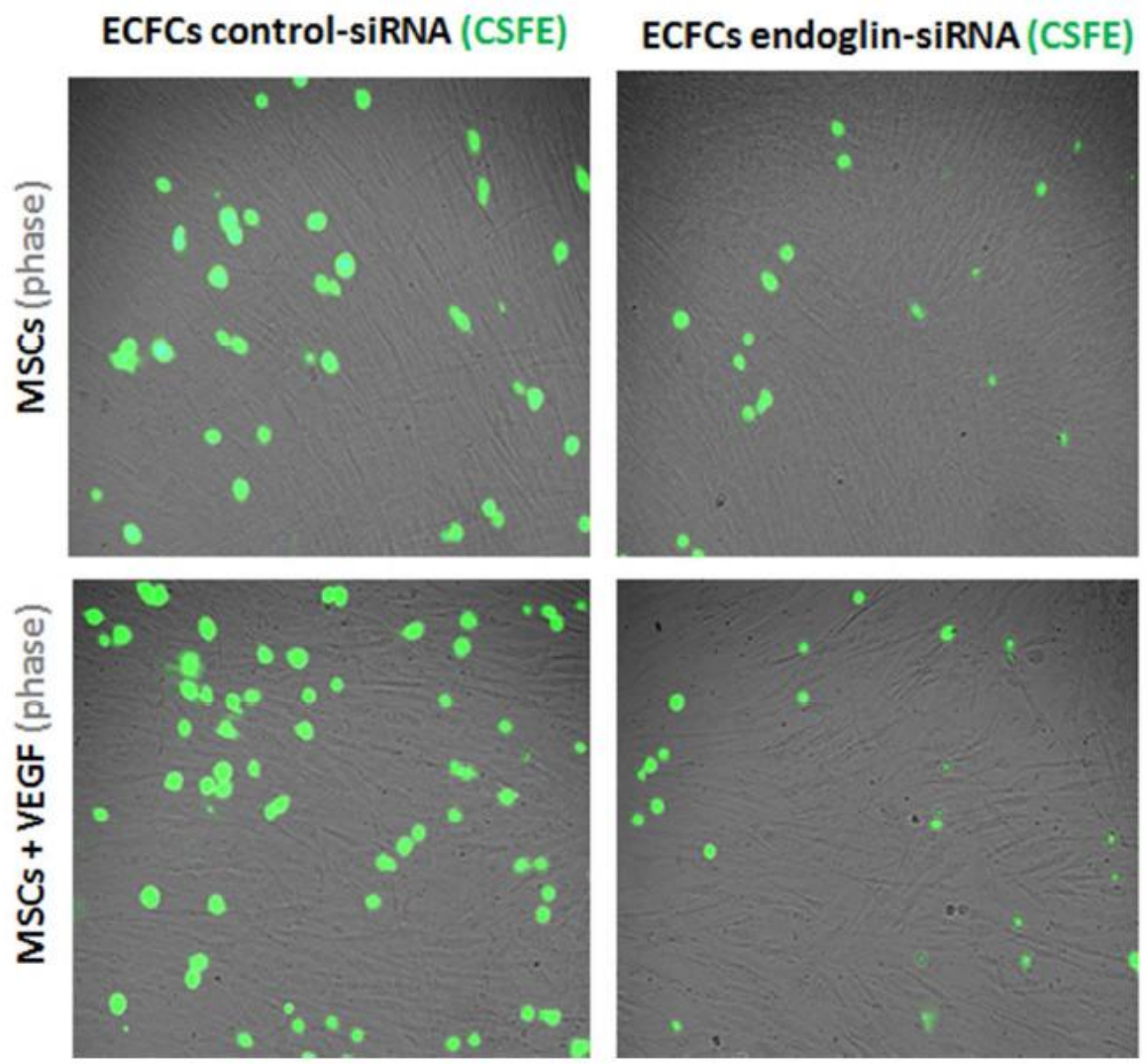

Figure 6A

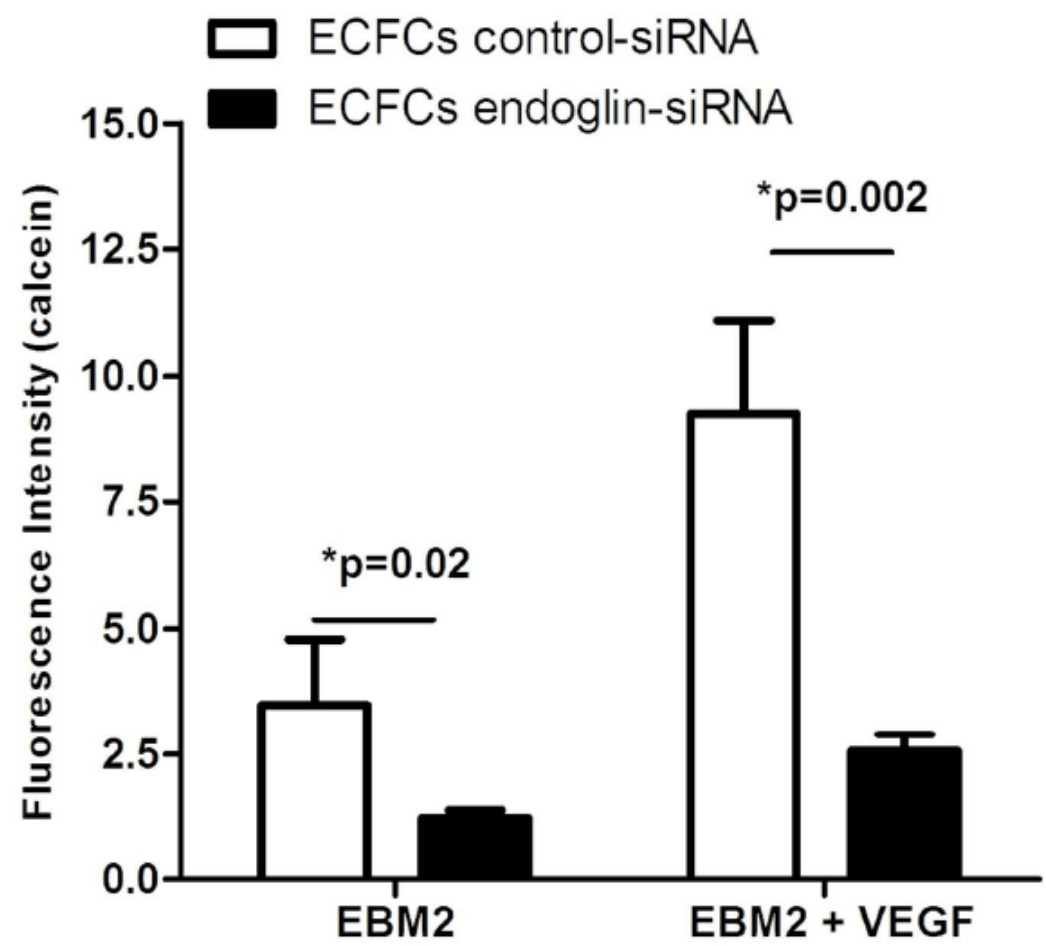

Figure 6B 